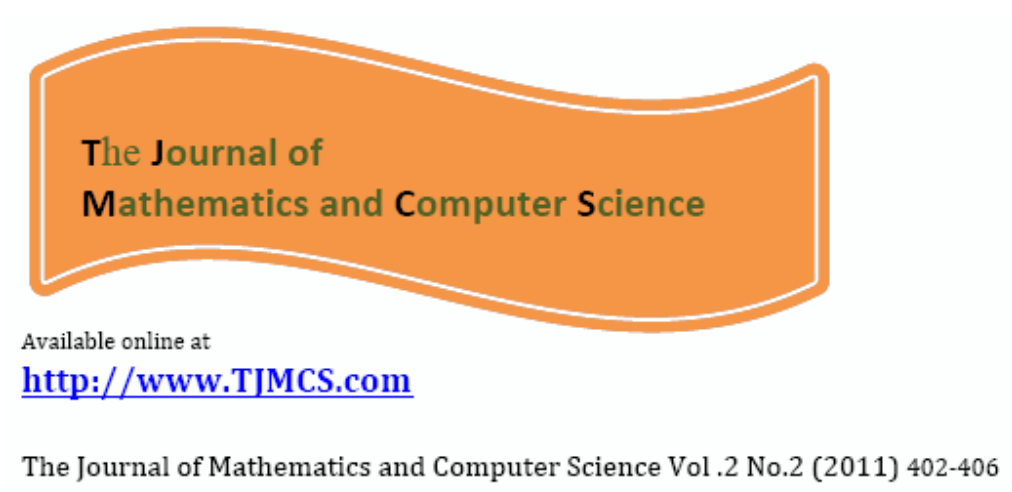

\title{
Commuting Graphs on Dihedral Group
}

\author{
T. Tamizh Chelvam , K. Selvakumar and S. Raja \\ Department of Mathematics, Manonmanian Sundaranar, \\ University Tirunelveli 627 012, Tamil Nadu, India \\ Tamche_59@yahoo.co.in, selva_158@yahoo.co.in, nellairajaa@yahoo.com
}

\begin{abstract}
A bstract
Let $\Gamma$ be a non-abelian group and $\Omega \subseteq \Gamma$. The commuting graph $\mathrm{C}(\Gamma, \Omega)$, has $\Omega$ as its vertex set with two distinct elements of $\Omega$ joined by an edge when they commute in $\Gamma$. In this paper we discuss certain properties of commuting graphs constructured on the dihedral group $\mathrm{D}_{2 n}$ with respect to some specific subsets. More specifically we obtain the chromatic number and clique number of these commuting graphs.
\end{abstract}

2000 Mathematics Subject Classification: 05C.

Keywords: Commuting graph, dihedral group, clique number, chromatic number, split graph

\section{Introduction}

The study of algebraic structures, using the properties of graphs, becomes an exciting research topic in the last twenty years, leading to many fascinating results and questions. For example, the study zero-divisor graphs, total graph of commutative rings and commuting graph of groups has attracted many researchers towards this dimension. One can refer [2, 3] for such studies. The concept of non-commuting graph has been studied in [1], where as the concept of commuting graph has been found in[4]. For basic definitions one can refer [5, 6]. For any integer $\mathrm{n} \geq 3$, the Dihedral group $2 \mathrm{n}$ is given by $\mathrm{D}_{2} \mathrm{n}=\left\langle\mathrm{r}, \mathrm{s}: \mathrm{s}^{2}=\right.$ $\mathbf{r}^{\mathbf{n}}=1, \mathrm{rs}=\mathrm{sr}^{-1}>$. For any subset $\Omega$ of $\mathrm{D}_{2} \mathbf{n}$, the commuting graph $\mathrm{C}\left(\mathrm{D}_{2} \mathbf{n}\right.$, $\Omega$ ) has $\Omega$ as its vertex set with two distinct vertices in $\Omega$ are adjacent if they commute with each other in $\mathrm{D}_{2} \mathbf{n}$.

The center of a group $\Gamma$ is denoted by $Z(\Gamma)$. Let $\Omega$ be any nonempty subset of $\Gamma$. The centralizer of $\Omega$ in $\Gamma$ is the set of elements of $\Gamma$ which commutes with every element of $\Omega$ and it is denoted by $\mathrm{C} \Omega(\Gamma)$.

We consider simple graphs which are undirected, with no loops or multiple edges.

For any graph $\mathrm{G}$, we denote the sets of the vertices and edges of $\mathrm{G}$ 
by $V(G)$ and $E(G)$, respectively. The degree $\operatorname{deg}_{G}(v)$ of a vertex $v$ in $G$ is the number of edges incident to $v$. The order of $G$ is defined $|V(G)|$ and its maximum and minimum degrees will be denoted, respectively, by $\Delta(G)$ and $\delta(G)$. A graph $G$ is regular if the degrees of all vertices of $G$ are the same. A subset $\Omega$ of the vertices of $G$ is called a clique if the induced subgraph of $\Omega$ is a complete graph. The maximum size of a clique in a graph $G$ is called the clique number of $G$ and denoted by $\omega(G)$. Let $k>0$ be an integer. A $k$-vertex coloring of a graph $G$ is an assignment of $k$ colors to the vertices of $G$ such that no two adjacent vertices have the same color. The chromatic number $\chi(G)$ of a graph $G$, is the minimum $k$ for which $G$ has a $k$-vertex coloring. If $u$ and $v$ are vertices in $G$, the $d(u, v)$ denotes the length of the shortest path between $u$ and $v$. The largest distance between all pairs of the vertices of $G$ is called the diameter of $G$, and is denoted by $\operatorname{diam}(G)$. A graph $G$ is defined to be split if there is a partition $V=S+K$ of its vertex set into a independent set $S$ and a complete set $K$.

\section{Main Results}

Throughout this section, $n \geq 3$ is an integer and $D_{2 n}=<r, s: s^{2}=r^{n}=1$, $r s=s r^{-1}>, \Omega_{1}=\left\{r^{1}, r^{2}, \ldots, r^{n}\right\}$ and $\Omega_{2}=\left\{s r^{1}, s r^{2}, \ldots, s r^{n}\right\}$ are subsets of $D_{2 n}$. In this section we obtain certain properties of the commuting graph constructed on the dihedral group $D_{2 n}$. More specifically, we discuss properties about commuting graphs constructed on $D_{2 n}$, with respect to $\Omega_{1}$ and $\Omega_{2}$.

Lemma 2.1. Let $\Omega$ be any subset of $D_{2 n}$ and let $G=\mathcal{C}\left(D_{2 n}, \Omega\right)$ be a commuting graph. Then for any $a \in \Omega$, $\operatorname{deg}_{G}(a)=\left|C_{\Omega}(a)\right|-1$.

Proof. Let $a \in \Omega$. Then $a \in C_{\Omega}(a), C_{\Omega}(a)$ is the set of elements in $\Omega$ which commutes with $a$ and hence $\operatorname{deg}(a)=\left|C_{\Omega}(a)\right|-1$.

Lemma 2.2. Let $n \geq 3$ be an even integer. Let $G=\mathcal{C}\left(D_{2 n}, D_{2 n}\right)$. Then

(i). $\operatorname{deg}_{G}\left(s r^{i}\right)=3$ for all $i, 1 \leq i \leq n$

(ii). $\operatorname{deg}_{G}\left(r^{i}\right)= \begin{cases}2 n-1 & \text { if } i=n, \frac{n}{2} \\ n-1 & \text { otherwise }\end{cases}$

Proof. (i) Since $C_{D_{2 n}}\left(s r^{i}\right)=\left\{e, r^{\frac{n}{2}}, s r^{i}, s r^{\frac{n}{2}+i}\right\}$ for all $i, 1 \leq i \leq n$, we can see that $\operatorname{deg}_{G}\left(s r^{i}\right)=3$ for all $i, 1 \leq i \leq n$.

(ii) Suppose $i=n, \frac{n}{2}$. Then $C_{D_{2 n}}\left(r^{i}\right)=D_{2 n}$ and so $\operatorname{deg}_{G}\left(r^{i}\right)=2 n-1$. If $i \neq n, \frac{n}{2}$, then $C_{D_{2 n}}\left(r^{i}\right)=\left\{r^{i}: 1 \leq i \leq n\right\}$ and so $\operatorname{deg}_{G}\left(r^{i}\right)=n-1$.

Lemma 2.3. Let $n \geq 3$ be an odd integer. Let $G=\mathcal{C}\left(D_{2 n}, D_{2 n}\right)$. Then

(i). $\operatorname{deg}_{G}\left(s r^{i}\right)=1$ for all $i, 1 \leq i \leq n$

(ii). $\operatorname{deg}_{G}\left(r^{i}\right)= \begin{cases}2 n-1 & \text { if } i=n \\ n-1 & \text { otherwise }\end{cases}$ 
Proof. (i) Since $C_{D_{2 n}}\left(s r^{i}\right)=\left\{e, s r^{i}\right\}$ for all $i, 1 \leq i \leq n$, it follows that $\operatorname{deg}_{G}\left(s r^{i}\right)=1$ for all $i, 1 \leq i \leq n$.

(ii) Suppose $i=n$. Then $C_{D_{2 n}}\left(r^{i}\right)=D_{2 n}$ and so $\operatorname{deg}_{G}\left(r^{i}\right)=2 n-1$. If $i \neq n$, then $C_{D_{2 n}}\left(r^{i}\right)=\left\{r^{i}: 1 \leq i \leq n\right\}$ and so $\operatorname{deg}\left(r^{i}\right)=n-1$.

Theorem 2.4. Let $G=\mathcal{C}\left(D_{2 n}, \Omega\right)$ be a commuting graph, where $\Omega$ is a subset of $D_{2 n}$ and $n \geq 3$. Then the following are true:

(i) If $\Omega$ is an abelian subgroup of $D_{2 n}$, then $\operatorname{diam}(G)=1$.

(ii) If $\Omega$ is a subgroup of $D_{2 n}$, then $\operatorname{diam}(G)=2$.

(iii) If $\Omega=D_{2 n}-Z\left(D_{2 n}\right)$, then $\operatorname{diam}(G)=\infty$.

Proof. (i) If $\Omega$ is an abelian subgroup of $D_{2 n}$, then $G$ is a complete graph and so $\operatorname{diam}(G)=1$.

(ii) Let $\Omega$ be a subgroup of $D_{2 n}$. Note that $G$ is connected. Since $\Omega$ is not abelian, $x y \neq y x$ for some $x, y \in \Omega$ and so $\operatorname{diam}(G) \geq 2$. Since $e \in \Omega, x-e-y$ is a path of length 2 and hence $\operatorname{diam}(G)=2$.

(iii) Let $\Omega=D_{2 n}-Z\left(D_{2 n}\right)$. Then $G$ is disconnected and so $\operatorname{diam}(G)=\infty$.

In view of Lemma 2.4, we have following corollary.

Corollary 2.5. Let $G=\mathcal{C}\left(D_{2 n}, \Omega\right)$, where $\Omega$ is a subset of $D_{2 n}$.

(i) If $n$ is odd and $\Omega=\left\{r^{i}\right.$, sr ${ }^{j}$ : for any $1 \leq i \leq n-1$ and $\left.1 \leq j \leq n\right\}$, then $\operatorname{diam}(G)=\infty$.

(ii) If $n$ is even and $\Omega=\left\{r^{i}\right.$, sr $r^{j}$ : for any $1 \leq i \leq n-1, i \neq \frac{n}{2}$ and $1 \leq$ $j \leq n\}$, then $\operatorname{diam}(G)=\infty$.

Theorem 2.6. Let $n \geq 3$ be an integer and $G=\mathcal{C}\left(D_{2 n}, \Omega\right)$, where $\Omega$ is a subset of $D_{2 n}$ and $n \geq 3$. Then $G=K_{n}$ if and only if $\Omega=\Omega_{1}$.

Proof. Suppose $\Omega=\left\{r^{i}: 1 \leq i \leq n\right\}$. Then $\Omega$ is a cyclic subgroup of $D_{2 n}$ and so $G$ is a complete graph on $n$ vertices. Conversely, suppose $G=K_{n}$. By Lemma 2.2 and Lemma 2.3, hence $\Omega=\left\{r^{1}, r^{2}, \ldots, r^{n}\right\}$.

By Lemmas 2.2 and 2.3, in any commuting graph $G=\mathcal{C}\left(D_{2 n}, \Omega\right)$, the degree of vertices $s r^{i}$ for $1 \leq i \leq n$ can be either 1 or 3 . Hence from the above Lemma 2.6, we have following corollary.

Corollary 2.7. Let $n \geq 3$. Then there exists no subset $\Omega$ of $D_{2 n}$ such that $\mathcal{C}\left(D_{2 n}, \Omega\right)$ is $n$ regular.

Theorem 2.8. Let $n \geq 3$ be an integer and $\mathcal{C}\left(D_{2 n}, \Omega\right)$, where $\Omega$ is any subset of $D_{2 n}$. If $n$ is odd, then $\mathcal{C}\left(D_{2 n}, \Omega\right)=K_{1, n}$ if and only if $\Omega=$ $\left\{e, s r^{1}, s r^{2}, \ldots, s r^{n}\right\}$.

Proof. Suppose $\Omega=\left\{e, s r^{1}, s r^{2}, \ldots, s r^{n}\right\}$. Then $C_{\Omega}\left(s r^{i}\right)=\{e\}$ and $C_{\Omega}(e)=\Omega$ and so $\mathcal{C}\left(D_{2 n}, \Omega\right)=K_{1, n}$. Conversely, suppose $\mathcal{C}\left(D_{2 n}, \Omega\right)=K_{1, n}$. Then by Lemma $2.3, \Omega=\left\{e, s r^{1}, s r^{2}, \ldots, s r^{n}\right\}$. 
Corollary 2.9. Let $n \geq 3$ be an odd integer and $\mathcal{C}\left(D_{2 n}, D_{2 n}\right)$. Then $G$ is split graph.

Proof. This proof follows from Theorem 2.6 and Theorem 2.8.

Theorem 2.10. For any integer $n \geq 2$, let $G=\mathcal{C}\left(D_{2 n}, D_{2 n}\right)$. Then the number of edges in $G, \epsilon(G)= \begin{cases}\frac{n(n+1)}{2} & \text { if } n \text { is odd } \\ \frac{n(n+4)}{2} & \text { otherwise. }\end{cases}$

Proof. Note that $\Omega_{1} \cap \Omega_{2}=\emptyset$ and $\Omega_{1} \cup \Omega_{2}=D_{2 n}$.

Case 1. Suppose $n$ is even. Clearly the subgraph induced by $\Omega_{1}$ is complete and the subgraph induced by $\Omega_{2}$ is $\frac{n}{2} K_{2}$. Therefore the number of edges in $G$ is sum of the number of edges in $\left\langle\Omega_{1}\right\rangle$, the number edges in $\left\langle\Omega_{2}\right\rangle$ and the number of edges from $\left\{r^{n}, r^{\frac{n}{2}}\right\}$ to set of vertices in $\Omega_{2}$. Thus $\epsilon(G)=$ $\frac{n(n-1)}{2}+\frac{n}{2}+2 n=\frac{n(n+4)}{2}$.

Case 2. Suppose $n$ is odd. In this case, the subgraph induced by $\Omega_{1}$ is complete and the subgraph induced by $\Omega_{2}$ has no edges. Therefore the number of edges in $G$ is sum of the number of edges in $\left\langle\Omega_{1}\right\rangle$ and the number of edges from $r^{n}$ to set of vertices in $\Omega_{2}$. Thus $\epsilon(G)=\frac{n(n-1)}{2}+n=\frac{n(n+1)}{2}$.

Theorem 2.11. Let $n \geq 3$ be any integer. Then there exists no subset $\Omega$ of $D_{2 n}$ such that $G=\mathcal{C}\left(D_{2 n}, \Omega\right)=C_{4}$.

Proof. Suppose $\mathcal{C}\left(D_{2 n}, \Omega\right)=C_{4}$ for some subset $\Omega$ of $D_{2 n}$. If $n$ is odd, then by Lemma $2.3, s r^{i} \notin \Omega$ for all $i, 1 \leq i \leq n$ and so $\Omega \subset \Omega_{1}$. From this $\mathcal{C}\left(D_{2 n}, \Omega\right)$ is an induced subgraph of $\mathcal{C}\left(D_{2 n}, \Omega_{1}\right)$. Since $\mathcal{C}\left(D_{2 n}, \Omega_{1}\right)$ is complete graph, $\mathcal{C}\left(D_{2 n}, \Omega\right)$ is complete, a contradiction.

Assume that $n$ is even. Then $\Omega$ contains at least two vertices from $\Omega_{2}$, otherwise it will contain at least three vertices from $\Omega_{1}$ and those vertices will induce at least $K_{3}$, a contradiction. Suppose $\Omega \subset \Omega_{2}$. Then every vertex in $\mathcal{C}\left(D_{2 n}, \Omega\right)$ is of degree 1 , a contradiction. Suppose $\Omega$ contains three vertices from $\Omega_{2}$. Then $\Omega$ contains one vertex from $\Omega_{1}$ and it could be either $e \in \Omega$ or $r^{\frac{n}{2}} \in \Omega$ and not both. In both the cases the degree of that vertex is 3 and hence we get a contradiction. Thus $\Omega$ contains two vertices from $\Omega_{2}$ and another two vertices from $\Omega_{1}$. From this we have $\Omega=\left\{e, r^{\frac{n}{2}}, s r^{i}, s r^{j}\right\}$ with $i \neq j$. From this we get that $\operatorname{deg}_{G}(e)=\operatorname{deg}_{G}\left(r^{\frac{n}{2}}\right)=3$, a contradiction.

As prove above, one can prove the following:

Lemma 2.12. Let $n \geq 3$ be any integer. Then there exists no subset $\Omega$ of $D_{2 n}$ such that $\mathcal{C}\left(D_{2 n}, \Omega\right)=P_{4}$.

Theorem 2.13. Let $n \geq 2$ be any integer and let $G=\mathcal{C}\left(D_{2 n}, D_{2 n}\right)$. Then $\omega(G)=\chi(G)=n$. 
Proof. Consider $\Omega_{1}=\left\{r^{1}, r^{2}, \ldots, r^{n}\right\} \subset D_{2 n}$. As observed earlier, $\mathcal{C}\left(D_{2 n}, \Omega_{1}\right)$ is a maximal complete subgraph of $G$. Hence $\omega(G)=n$.

Case 1. If $n$ is even, then one needs $n$ colors to color $<\Omega_{1}>\subset G$ and so $\chi(G) \geq n$. Note that $e$ and $r^{\frac{n}{2}}$ are adjacent to all vertices and so colors assigned to theses vertices cannot be assigned to any other vertex. e given to any other vertices. The remaining $n-2$ vertices in $\Omega_{1}$ are not adjacent to any of the remaining vertices in $\mathcal{C}\left(D_{2 n}, D_{2 n}\right)-\Omega_{1}$ and so these vertices can be colored one of the remaining $n-2$ colors. Hence $\chi(\mathcal{C}(G, \Omega))=n$.

Case 2. When $n$ is odd, one can use the colors of $r^{i}$ except $e=r^{n}$ to the vertices in $\Omega_{2}$ and hence the proof follows.

Theorem 2.14. Let $n \geq 2$ be any even integer. Then $G=\mathcal{C}\left(D_{2 n}, D_{2 n}\right)$ has a perfect matching.

Proof. Since $n$ is even as observed in the proof of Lemma 2.10, $\left\langle\Omega_{1}\right\rangle$ is a complete subgraph of $G$ and $\left\langle\Omega_{2}\right\rangle=\frac{n}{2} K_{2}$, where $\Omega_{1}=\left\{r^{1}, r^{2}, \ldots, r^{n}\right\}$ and $\Omega_{2}=$ $\left\{s r^{1}, s r^{2}, \ldots, s r^{n}\right\}$. Since $n$ is even, $\left\langle\Omega_{1}\right\rangle$ and $\left\langle\Omega_{2}\right\rangle$ have perfect matchings.

Corollary 2.15. Let $n \geq 2$ be any even integer and $n=2 k$. Then number of perfects matchings in $G=\mathcal{C}\left(D_{2 n}, D_{2 n}\right)$ is $\frac{n !}{2^{n} k !}+1$.

Proof. As in the proof of Lemma 2.14, the number of perfect matchings in $\langle\Omega\rangle$ is $\frac{n !}{2^{n} k !}$ and the number of perfect matchings in $\langle Y\rangle$ is 1 . Hence the number of perfect matching in $G$ is $\frac{n !}{2^{n} k !}+1$.

\section{References}

[1] A. Abdollaho, S. Akbari and H.R. Maimani, Non-commuting graph of a group, J. of Algebra, 298(2006), 468-492.

[2] D.F. Anderson and P. Livingston, The zero-divisor graph of a commutative ring, J. of Algebra 217(1999), 434-447.

[3] D.F. Anderson and Ayman Badawi, The total graph of a commutative ring, J. of Algebra, 320(2008), 2706-2719.

[4] D.Bundy, The Connectivity of Commuting Graphs, J. Comb. Theory, Ser. A 113, Issue 6(2006), 995-1007.

[5] David S. Dummit and Richard M.Foote, Abstract Algebra (Second Edition), John Wiley and Son, Inc(Asia) Pvt. Ltd, Singapore(2005).

[6] F. Harary, Graph Theory, Addision-Wesley Reading M.A, 1969. 